\title{
La linguistique française à La Nouvelle-Orléans : un état des lieux 2010
}

\author{
Jacques Durand \\ CLLE (Cognition, Langues, Langage, Ergonomie, CNRS), Toulouse Le Mirail, Institut Universitaire de France \\ Thomas Klingler \\ Université Tulane, La Nouvelle- Orléans \\ Lorenza Mondada \\ ICAR (Interactions, Corpus, Apprentissages, Représentations, CNRS), Université Lumière Lyon 2 \\ Valelia Muni Toke \\ Histoire des théories linguistiques (HTL, CNRS) \\ Franck Neveu \\ ILF (Institut de Linguistique Française), CRISCO (Centre de Recherches Inter-langues sur la Signification en \\ Contexte), Université de Caen \\ Sophie Prévost \\ Langues, Textes, Traitements informatiques, Cognition (LaTTiCe, CNRS)
}

\section{La linguistique française 2010 : un état des lieux}

Jacques Durand, Benoît Habert et Bernard Laks (2008), dans leur introduction aux actes du Congrès de 2008, notaient que la linguistique française était éparpillée et qu'il n'existait pas de manifestation globale permettant d'en connaître en temps réel les avancées et les points d'ombre. L'Institut de Linguistique Française (ILF - présenté à la suite de cette introduction des actes) et ses partenaires ont organisé avec succès à Paris la première édition d'une telle manifestation, le Congrès Mondial de Linguistique Française (CMLF-08).

Le premier Congrès Mondial de 2008 a présenté un état de la recherche sur le français sous la forme de 8 conférences transversales invitées et de 150 communications orales réparties en douze thématiques. Dès l'ouverture du Congrès, ces conférences et communications ont été disponibles aux participants sous la forme d'un livret de résumés, d'un CD-ROM contenant les contributions et de leur mise en ligne sur le site de l'ILF. Le succès du CMLF-2008 et l'écho de cette manifestation nous a incités à pérenniser cette manifestation. La deuxième édition a été organisée à La Nouvelle-Orléans, aux États-Unis.

\section{Le choix de La Nouvelle-Orléans}

Le titre « Congrès Mondial de Linguistique Française » a pu paraître à la fois hyperbolique, évoquant les événements sportifs à l'échelle planétaire et trop complice d'une logique mondialisante que dénoncent de nombreux collègues, voire de nombreux travaux sur la variation sociolinguistique. Nous sommes sensibles à leur inquiétude, mais, de fait, la recherche en linguistique française ne se fait plus en vase clos depuis fort longtemps et l'évaluation de la recherche dans toutes les disciplines tient compte de l'impact des avancées sur le plan international. Les spécialistes de linguistique française (de la phonétique à l'étude du discours) participent tous à des colloques internationaux dont certains migrent de pays en pays de façon régulière. Ce qui manquait à la communauté était un forum international où toutes les recherches 
sur la langue française ont droit de cité et dont les résultats sont accessibles de façon immédiate. Alors qu'il existe déjà des congrès internationaux de linguistique française, le terme de congrès mondial veut souligner la perspective de rassemblement de la communauté au-delà des thématiques ou des disciplines, et montrer la vivacité des travaux en linguistique française bien au-delà de la France et des pays francophones. Après le choix de la capitale française pour des raisons pratiques lors du premier CMLF, il était important de signaler que la linguistique française n'est pas uniquement faite en France, et, incidemment, que la langue française n'appartient pas seulement à l'élite intellectuelle ou sociale du vieil hexagone. Le choix d'une partie du monde où le français a été minoré au cours de l'histoire véhicule pour nous un message d'ouverture vers toutes ces communautés où le français et son étude doivent parfois lutter pour leur survie. Nous remercions donc l'Université Tulane pour son accueil et son soutien dans l'organisation du CMLF 2010.

Le prochain CMLF aura lieu en 2012 à Lyon, où le laboratoire ICAR - Interactions, Corpus, Apprentissages, Représentations - se fera le relais local de l'ILF. Il est déjà prévu que l'édition suivante quittera à nouveau la France.

\section{Thématiques, auteurs, soumissions}

Le CMLF 2010 est organisé en 13 sessions dont 12 thématiques et six grandes conférences par invitation. Ces thématiques sont sensiblement les mêmes que lors du précédent congrès :

- 1 - Diachronie

- 2 - Didactique et enseignement, français langue maternelle, français langue seconde

- 3 - Discours, pragmatique et interaction

- 4 - Histoire, épistémologie, réflexivité

- 5 - Lexique et morphologie

- 6 - Linguistique du texte et de l'écrit, stylistique

- 7 - Phonétique, phonologie et interfaces

- 8 - Psycholinguistique et acquisition

• 9 - Sémantique

- 10 - Sociolinguistique et écologie des langues

-11 - Syntaxe

- 12 - Traitement automatique des langues

Les participants pouvaient aussi soumettre des articles « hors thématiques».

A la différence du CMLF 2008, les coordonnateurs des thématiques n'ont rédigé pour cette édition aucun texte d'orientation sur le contenu des recherches. Les textes d'orientation en 2008 visaient à suggérer des pistes possibles de recherche, mais ils ont été parfois perçus comme normatifs et interprétés comme des programmes visant à définir ce qui était « recevable» dans tel ou tel champ ou comme visant à restreindre ces champs de manière arbitraire. Il nous a donc semblé que des labels disciplinaires plus ou moins traditionnels suffisaient à orienter les auteurs de communication. Nous avons en outre ouvert la possibilité de travailler "hors thématiques », c'est-à-dire en marge des sous-disciplines traditionnelles ou bien de manière pluri-thématique. Toutefois, très peu de soumissions ont retenu cette option, et, généralement, 
toutes les propositions se sont rattachées naturellement à telle ou telle sous-discipline. Nous avons néanmoins respecté les vœux des auteurs et organisé une treizième session pour les chercheurs qui considèrent leur travail comme se positionnant ailleurs que dans les périmètres disciplinaires traditionnels.

Quelques données quantitatives relatives au CMLF 2010 :

\section{Auteurs / Communications}

\begin{tabular}{|l|c|}
\hline $\begin{array}{l}\text { Nombre de propositions de communication déposées } \\
\text { sur la plateforme }\end{array}$ & 261 \\
\hline $\begin{array}{l}\text { Nombre total d'auteurs ayant proposé une } \\
\text { communication }\end{array}$ & 295 \\
\hline Nombre de communications acceptées & $152=58,23 \%$ \\
\hline Nombre de communications refusées & $109=41,76 \%$ \\
\hline $\begin{array}{l}\text { Nombres total d'auteurs concernés par les } \\
\text { communications acceptées }\end{array}$ & 225 \\
\hline $\begin{array}{l}\text { Nombre de jeunes chercheurs (doctorants ou docteurs } \\
\text { depuis moins de } 3 \text { ans) concernés par les } \\
\text { communications acceptées }\end{array}$ & $59=26,22 \%$ \\
\hline
\end{tabular}

Répartition géographique des auteurs concernés par les communications acceptées

\begin{tabular}{|l|c|}
\hline France & 139 \\
\hline USA & 18 \\
\hline Canada & 16 \\
\hline Suisse & 10 \\
\hline Belgique & 9 \\
\hline Espagne & 4 \\
\hline Allemagne & 3 \\
\hline Angleterre & 3 \\
\hline Japon & 3 \\
\hline Norvège & 3 \\
\hline Algérie & 2 \\
\hline
\end{tabular}




\begin{tabular}{|l|c|}
\hline Israël & 2 \\
\hline Italie & 2 \\
\hline Maroc & 2 \\
\hline Vietnam & 2 \\
\hline Australie & 1 \\
\hline Brésil & 1 \\
\hline Congo & 1 \\
\hline Côte d'Ivoire & 1 \\
\hline Mexique & 1 \\
\hline Pologne & 1 \\
\hline Russie & 1 \\
\hline
\end{tabular}

\section{Les supports éditoriaux du Congrès}

Comme pour la première édition du CMLF, le comité d'organisation du Congrès ainsi que l'ensemble des coordonnateurs de thématiques ont souhaité que les actes soient disponibles à l'ouverture de la manifestation (livret des résumés, et $\mathrm{CD}$ regroupant l'ensemble des communications en version intégrale $\left.^{1}\right)$. La version électronique des actes est mise en ligne sur le site du Congrès (http://www.linguistiquefrancaise.org). La publication des articles en version intégrale (textes de 10 à 20 pages) ne constitue en aucun cas un obstacle à une publication ultérieure remaniée dans d'autres supports (revues, ouvrages collectifs ou personnels). Elle est une opportunité pour les chercheurs de proposer en temps réel un état de leur travail qui peut se prêter à toute forme d'évolution rapide par le biais des échanges.

\section{$5 \quad$ Le CMLF : un effort collectif}

Le CMLF représente un investissement collectif considérable. Son organisation n'est pas le seul fruit du travail du comité d'organisation qui cosigne cette préface. Le CMLF représente d'abord la prise en charge du Congrès par toutes les unités de recherche dont se compose l'Institut de Linguistique Française. Les directeurs de ces équipes de recherche (ou leurs représentants) ont tous œuvré pour le succès de cette entreprise en planifiant son organisation générale (notamment la constitution des comités d'organisation des différentes sessions) et en soutenant financièrement le montage du Congrès. A leur tour, les Présidents et Vice-Présidents des sessions ont dû mettre en place des comités scientifiques qui ont évalué les propositions en s'appuyant également sur des relecteurs issus de la communauté universitaire et scientifique des sciences du langage. Chaque soumission anonymisée a été évaluée par un minimum de deux lecteurs, en double aveugle. Si le système n'est pas parfait, il s'efforce de mettre sur un pied d'égalité toutes les propositions et de les faire évaluer qualitativement par les spécilaistes du domaine. C'est l'engagement que nous avions pris lors du lancement de ce projet.

Il convient d'insister sur le fait que l'évaluation porte non pas sur une intention de développement scientifique mais sur l'ensemble quasi finalisé de l'argumentation et de l'analyse. Cette exigence de 
rigueur peut sembler dissuasive tant est lourde la soumission à un congrès d'un texte d'une douzaine de pages. Mais l'édition 2010 du CMLF nous a permis de constater la familiarisation de la communauté des sciences du langage avec ce principe de soumission, qui permet notamment d'éviter la distorsion entre les propositions soumises qui ont été retenues et les communications effectivement prononcées auxquelles elles donnent lieu. L'engagement du chercheur dans sa participation à la manifestation scientifique se trouve ainsi renforcé, tout comme la portée et l'intérêt de l'évaluation.

Nous souhaitons pour finir adresser nos vifs remerciements aux organismes, institutions et personnels qui suivent, sans lesquels le CMLF 2010 n'aurait pu être réalisé à la hauteur de l'ambition qui a présidé à la création de la manifestation: l'Institut des Sciences Humaines et Sociales du CNRS, le Ministère de l'Enseignement Supérieur et de la Recherche, le Ministère de la Culture et la Délégation Générale à la Langue Française et aux Langues de France, l'Université Tulane (Nouvelle-Orléans), l'Agence Universitaire de la Francophonie, l'ensemble des unités composant l'ILF', les Présidents et VicePrésidents des thématiques du Congrès ainsi que les membres des comités scientifiques et les relecteurs, et bien sûr le secrétariat et le secrétariat général de l'ILF $^{3}$.

Le comité d'organisation du CMLF 2010

Jacques DURAND, directeur du laboratoire CLLE (Cognition, Langues, Langage, Ergonomie, CNRS), Toulouse Le Mirail, Institut Universitaire de France

Thomas KLINGLER, directeur du département de français de l'Université Tulane de la NouvelleOrléans

Lorenza MONDADA, directrice du laboratoire ICAR (Interactions, Corpus, Apprentissages, Représentations, CNRS), Université Lumière Lyon 2

Valelia MUNI TOKE, laboratoire d'Histoire des théories linguistiques (HTL, CNRS)

Franck NEVEU, directeur de l'ILF (Institut de Linguistique Française), directeur du CRISCO (Centre de Recherches Inter-langues sur la Signification en Contexte), Université de Caen

Sophie PREVOST, laboratoire Langues, Textes, Traitements informatiques, Cognition (LaTTiCe, CNRS)

\footnotetext{
${ }^{1}$ A partir des données fournies par la plateforme de la société Intellagence (http://congres.intellagence.eu), l'éditeur EDP Sciences (http://www.edpsciences.org) prend en charge la mise en ligne des actes du Congrès, l'édition du volume imprimé des résumés et la réalisation du CD-ROM.

${ }^{2}$ Voir plus loin le texte intitulé « L'Institut de Linguistique Française (CNRS, FR 2393) ».

${ }^{3}$ Voir plus loin, la composition des comités.
} 